\title{
Study of Charge Density Distributions and Elastic Charge Form Factors for ${ }^{40} \mathrm{Ca}$ and ${ }^{48} \mathrm{Ca}$
}

\author{
Arkan R. Ridha \\ Department of Physics, College of Science, University of Baghdad, Baghdad-Iraq. \\ Corresponding Author: arkan_rifaah@yahoo.com.
}

\begin{abstract}
The ground charge density distributions (CDD), elastic charge form factors and proton, charge, neutron, and matter root mean square $(r m s)$ radii for stable ${ }^{40} \mathrm{Ca}$ and ${ }^{48} \mathrm{Ca}$ have been calculated using single-particle radial wave functions of Woods-Saxon (WS) and harmonic-oscillator (HO) potentials. Different central potential depths are used for each subshell which is adjusted so as to reproduce the experimental single-nucleon binding energies. An excellent agreement between the calculated $\mathrm{rms}$ charge radii and experimental data are found for both nuclei using WS and HO potentials. The calculated proton $r m s$ radii for ${ }^{40} \mathrm{Ca}$ are found to be in good agreement with experiment data using both WS and $\mathrm{HO}$ potentials while the results for ${ }^{48} \mathrm{Ca}$ showed an overestimation in WS potential and slight overestimation in HO potential. The calculated neutron rms radii are found to be well predicted in $\mathrm{HO}$ potential for both ${ }^{40} \mathrm{Ca}$ and ${ }^{48} \mathrm{Ca}$, while there is overestimation in WS results for both isotopes. The calculated $\mathrm{rms}$ matter radii showed good agreement with experimental data for ${ }^{40} \mathrm{Ca}$ using WS potential while the result is underestimated in $\mathrm{HO}$ potential. For ${ }^{48} \mathrm{Ca}$, the results obtained with $\mathrm{HO}$ potential is underestimated and slightly underestimated with WS potential. For both nuclei, the calculated ground charge density distributions evaluated with WS are in better agreement with the data than those of HO potential. Finally, the results of the calculated elastic charge form factors demonstrate excellent agreement with experimental data for both nuclei under study in WS potential on contrary to the results of HO potential which are completely failed to predict the existence of third diffraction minimum.
\end{abstract}

[DOI: 10.22401/JNUS.20.3.13]

PACS number(s): 21.60.Cs, 25.30.Bf

Keywords: stable nuclei, ground density distribution, elastic form factor, root-mean-square radii, Woods-Saxon potential.

\section{Introduction}

The spatial extent of atomic nuclei and the radial distribution of nuclear charge and matter have received great attention [1]. They are important to explore sizes and shapes of nuclei, besides to test the validity of the nuclear single-particle wave functions used especially in density folding models [2]. Because of the Gaussian fall-off behavior at large $r$ of the harmonic-oscillator (HO) radial wave functions which does not reproduce the correct exponential tail. The $\mathrm{HO}$ potential is not accurate to describe the nuclear central confining potential because the potential continues to give a contribution even for much larger $r$ and does not become or approaches zero. Elton and Swift [3] generated wave functions in a parameterized single-particle local potential and adjusted the parameters so as to fit the shape of the wave functions to elastic electron scattering data and the eigenenergies to the proton separation energies in the $1 p$ and $2 s-1 d$ shell nuclei. Gibson et. al. [4] studied the ground state of the ${ }^{4} \mathrm{He}$ nucleus using the single-particle phenomenological model. Wave functions were generated from a potential (WS form) whose parameters are chosen to reproduce the correct neutron separation energy. The proton separation energy, electron scattering form factors were then calculated. Gamba et. al. [5] determined the parameters of a WS potential well for ten p-shell nuclei by fitting the electron scattering form factors and single-particle binding energies. Brown et. al. [6] described a new method of calculating nuclear charge and matter distributions. The method was applied to the core nuclei ${ }^{16} \mathrm{O}$ and ${ }^{40} \mathrm{Ca}$. Brown et. al. [7] calculated the rms radii of valence orbits in the tin isotopes using the single-particle potential model. Streets et. al. [8] extracted the nuclear matter distributions from high-energy proton scattering data for many nuclei and compared with calculations using the single- 
particle potential method with a standard potential. Brown et. al [9] analyzed the best available data on the charge and matter distributions of ${ }^{208} \mathrm{~Pb}$ using (a) a WS potential and (b) a Hartree-Fock potential based on Skyrme interactions (c) a combination of the two, WS for the surface region and SkyrmeHartree-Fock for the interior. Lojewski et. al. [10] used realistic single-particle WS potential to evaluate the mean-square charge radii of even-even nuclei. Lojewski and Dudek [11] evaluated the proton and neutron separation energies and mean square charge radii within the WS plus BCS model for even-even nuclei with $40 \leq A \leq 256$. The various parametrizations of the WS potential were examined. Schwierz et. al. [12] established a new parametrization for the WS potential. Its six parameters are fitted to single-particle spectra around doubly magic nuclides and experimental charge radii. In [13] the eigenvalues have been calculated using Numerov method for a Sturm-Liouville problem defined with the boundary values $R(0)=R(12)=0$. Recently, Arkan [14] has been calculated the nuclear charge density distributions, elastic charge form factors and rms charge, proton, neutron and matter radii for ${ }^{4} \mathrm{He},{ }^{12} \mathrm{C}$ and ${ }^{16} \mathrm{O}$ nuclei using WS and $\mathrm{HO}$ radial waves function using different well potential depths for WS potential for each subshell.

This work is an extension to the work in [12] and it is dedicated to calculate ground CDDS, elastic charge form factors and $\mathrm{rms}$ charge proton, neutron and matter radii of ${ }^{40} \mathrm{Ca}$ and ${ }^{48} \mathrm{Ca}$ nuclei using the radial wave functions of WS and $\mathrm{HO}$ potentials.

\section{Theoretical formulations}

The radial part of the Schrödinger equation for the single-particle radial wave function can be written as [6]:

$\left(\frac{\hbar^{2}}{2 \mu} \frac{d^{2}}{d r^{2}}-v(r)-\frac{l(l+1) \hbar^{2}}{2 \mu r^{2}}+\varepsilon_{n l j}\right) R_{n l j t_{z}}(r)=0$

where $\mu=m(A-1) / A$ is the reduced mass of the core $(A-1)$ and single nucleon, $m$ is the nucleon mass, $A$ is the atomic mass, $\varepsilon_{n l j}$ is the single nucleon binding energy, $R_{n l j}(r)$ is the radial eigenfunction of WS potential, $n, l$, and $j$ are the principal, orbital angular, and total quantum numbers.

In eq. (1), the local potential $v(r)$ can be written for WS potential as $[3,5]$ :

$v(r)=v_{\text {cent }}(r)+v_{\text {s.o. }}(r)+v_{c}(r)$

Where

$v_{\text {cent }}(r)=\frac{-U_{0}}{\left(1+e^{\left(\frac{r-R}{a}\right)}\right)}$

Represents the central part of $v(r), U_{0}$ is the strength or depth of central potential, the $a_{0}$ is the diffuseness and $R=r_{0}(A-1)^{1 / 3}$ is the radius parameter.

$v_{\text {s.o. }}(r)=$

$-2\left(\frac{\hbar}{m_{\pi} c}\right)^{2} \frac{U_{\text {s.o. }}}{r} \frac{d}{d r} \frac{1}{\left(1+e^{\left(\frac{1-R_{\text {S.o. }}}{a_{\text {s.o. }}}\right)}\right)}\langle\hat{l} . \hat{\sigma}\rangle=$

$2\left(\frac{\hbar}{m_{\pi} c}\right)^{2} \frac{U_{\text {S.o. }}}{r} \frac{e^{\left(\frac{1-R_{\text {S.o. }}}{a_{\text {S.o. }}}\right)}}{\left(1+e^{\left(\frac{1-R_{\text {S.o. }}}{a_{\text {S.o. }}}\right)}\right)^{2}}\langle\hat{l} . \hat{\sigma}\rangle$

where $\left(\frac{\hbar c}{m_{\pi} c^{2}}\right)^{2} \approx 2.0 \mathrm{fm}^{2}$ with $m_{\pi} c^{2}=$ $139.567 \mathrm{MeV}$ and $\hbar c=197.329 \mathrm{MeV} . \mathrm{fm}^{2}$.

$\langle\hat{l} . \hat{\sigma}\rangle=\left\{\begin{array}{c}-\frac{1}{2}(l+1) \quad \text { for } j=l-\frac{1}{2} \\ \frac{1}{2} l \quad \text { for } j=l+\frac{1}{2}\end{array}\right.$

Eq. (4) represents the spin-orbit part of $v(r), m_{\pi}$ is the pion mass, $U_{\text {s.o. }}$ is the strength or depth of spin-orbit potential, $a_{\text {s.o. }}$ is the diffuseness of spin-orbit part, $R_{\text {S.o. }}=r_{\text {S.O. }}(A-1)^{1 / 3}$ is the radius parameter of spin-orbit and $\hat{l}$ and $\hat{\sigma}$ are the angular momentum and the spin operators respectively.

Finally, in Eq. (2) $v_{c}(r)$ indicates the Coulomb potential generated by a homogeneous charged sphere and can be written as [15]:

$v_{C}(r)=\left\{\begin{array}{cc}(Z-1) \frac{e^{2}}{r} & \text { if } r>R \\ \frac{(Z-1) e^{2}}{2 R}\left[3-\frac{r^{2}}{R^{2}}\right] & \text { if } r<R\end{array}\right.$,

for protons and $v_{C}(r)=0$ for neutrons, with $e^{2}=1.44 \mathrm{MeV}$. fm.

Therefore, Eq. (2) can be written as: 
$v(r)=\frac{-U_{0}}{\left(1+e^{\left(\frac{r-R}{a}\right)}\right)}+\left(\frac{\hbar}{m_{\pi}}\right)^{2} \frac{1}{r} \frac{U_{\text {S.o. }}}{a_{\text {S.o. }}} \frac{e^{\left(\frac{r-R_{\text {S.O. }}}{a_{\text {S.o. }}}\right)}}{\left(1+e^{\left.\left(\frac{r-R_{S . O}}{a_{\text {S.o. }}}\right)\right)^{2}}\right.}\langle\hat{l} . \widehat{\sigma}\rangle+v_{C}(r)$

The point density distributions of neutrons and protons can be found from [16]:

$\rho_{t_{z}}(r)=\frac{1}{4 \pi} \sum_{n l j} X_{t_{z}}^{n l j}\left|R_{n l j t_{z}}(r)\right|^{2}$

where $t_{z}=1 / 2$ for proton, and $t_{z}=-1 / 2$ for neutron, $X_{t_{z}}^{n l j}$ represents the number of neutrons $\left(t_{z}=1 / 2\right)$ or protons $\left(t_{z}=1 / 2\right)$ in the $n l j$-subshell.

The matter density distributions can be calculated from Eq. (7) as

$\rho_{m}(r)=\rho_{t_{z}=1 / 2}(r)+\rho_{t_{z=-1 / 2}}(r)$.

It is worth mentioning that the summation in Eq. (7) and (8) spans all occupied orbits.

In order to compare the calculated point proton density distributions with the experimental densities, the finite proton size is required to be included. The charge density distribution $\rho_{c h}(r)$ (CDD) is obtained by folding the proton density $\rho_{p r}$ into the distribution of the point proton density in Eq. (7) as follows [17]:

$\rho_{c h}(r)=\int \rho_{p}(r) \rho_{p r}\left(\boldsymbol{r}-\boldsymbol{r}^{\prime}\right) d \boldsymbol{r}^{\prime}$

If $\rho_{p}(\vec{r})$ is taken to have a Gaussian form, then

$\rho_{p r}(r)=\frac{1}{\left(\sqrt{\pi} a_{p r}\right)^{3}} e^{\left(\frac{-r^{2}}{a_{p r}^{2}}\right)}$

where $a_{p r}=0.65 \mathrm{fm}$. Such value of $a_{p r}$ reproduces the experimental charge $r m s$ radius of the proton, $\left\langle r^{2}\right\rangle_{p r}^{1 / 2}=\left(\frac{3}{2}\right)^{1 / 2} a_{p r} \approx$ $0.8 \mathrm{fm}$.

The rms radii of neutron, proton, charge and matter can be directly deduced from their density distributions [17] as follows:

$$
\left\langle r^{2}\right\rangle_{n, p, c h, m}^{1 / 2}=\sqrt{\frac{4 \pi}{X} \int_{0}^{\infty} \rho_{n, p, c h, m}(r) r^{2} d r} \ldots
$$

where $X$ stands for $N$ (number of neutrons), $Z$ (atomic number which is the same for proton and charge) and $A$, respectively.
In the first Born approximation the elastic neutron, proton, charge and matter form factors are Fourier transforms of the corresponding density distributions [17]:

$F_{n, p, c h, m}(q)=\frac{4 \pi}{q X} \int_{0}^{\infty} \rho_{n, p, c h, m}(r) \sin (q r) r d r$

Finally, the HO radial wave function used to calculate the neutrons, protons, charge and matter rms radii, CDDs, elastic charge form factors can be written as [18]:

$$
\begin{gathered}
R_{n l}\left(r, b_{t_{z}}\right)=\frac{1}{(2 l+1) ! !}\left[\frac{2^{l-n+3}(2 n+2 l-1) ! !}{\sqrt{\pi} b_{t_{z}}{ }^{3}(n-1) !}\right]^{\frac{1}{2}} \\
\times\left(\frac{r}{b_{t_{z}}}\right)^{l} e^{-\frac{r^{2}}{2 b_{t_{z}}{ }^{2}}}{ }_{1} F_{1}\left(1-n, l+\frac{3}{2} ; \frac{r^{2}}{b_{t_{z}}{ }^{2}}\right) \ldots \ldots \ldots \ldots \ldots . .(13)
\end{gathered}
$$

Where ${ }_{1} F_{1}\left(1-n, l+\frac{3}{2} ; \frac{r^{2}}{b_{t_{z}}{ }^{2}}\right)$ is the confluent hypergeometric function and is given by:

$$
\begin{aligned}
& { }_{1} F_{1}\left(1-n, l+\frac{3}{2} ; \frac{r^{2}}{b_{t_{z}}{ }^{2}}\right)= \\
& \sum_{k=0}^{n-1}(-1)^{k} \frac{(n-1) ! 2^{k}(2 l+1) ! !}{(n-k-1) ! k !(2 l+2 k+1) ! !}\left(\frac{r}{b_{t_{z}}}\right)^{2 k}
\end{aligned}
$$

\section{Results and Discussion:}

The nuclear CDDs, elastic charge form factors, and rms charge, proton, neutron and matter radii are calculated in terms of the independent particle model $[15,16]$ for ${ }^{40} \mathrm{Ca}$ and ${ }^{48} \mathrm{Ca}$. Two central potential are used to generate the single-nucleon radial wave function; WS and $\mathrm{HO}$ potentials. The experimental single-nucleon binding energies are used to regenerate the parameters of WS potential for each subshell; $U_{0}, U_{\text {s.o. }}, a_{0}, a_{\text {s.o. }}$, $r_{0}, r_{S . O}$, and $R_{C}$ for the nuclei under study.

For ${ }^{40} \mathrm{Ca}$ and ${ }^{48} \mathrm{Ca}$ nuclei, the chosen parameters for the WS potential are presented in Table (1) and (2) for ${ }^{40} \mathrm{Ca}$ and ${ }^{48} \mathrm{Ca}$ nuclei, respectively.

The regenerated single-nucleon binding energies are tabulated in Tables (3) and 4 for ${ }^{40} \mathrm{Ca}$ and ${ }^{48} \mathrm{Ca}$ nuclei, respectively. 
Table (1)

The WS parameters $U_{0}, U_{\text {s.o. }}, a_{0}, a_{\text {s.o }}, r_{0}, r_{S . o}$, and $R_{C}$ for ${ }^{40}$ Ca nucleus.

\begin{tabular}{||c||c||c|c|c|c||c||c||c||}
\hline${ }^{40} \mathbf{C a}$ & $\boldsymbol{n l}_{\boldsymbol{j}}$ & $\boldsymbol{U}_{\mathbf{0}}$ & $\boldsymbol{U}_{\text {s.o. }}$ & $\boldsymbol{a}_{\mathbf{0}}$ & $\boldsymbol{a}_{\text {s.o. }}$ & $\boldsymbol{r}_{\mathbf{0}}$ & $\boldsymbol{r}_{\text {S.o. }}$ & $\boldsymbol{R}_{\boldsymbol{C}}$ \\
\hline \hline \multirow{5}{*}{$n$} & $1 s_{1 / 2}$ & 52.724 & 15.0 & 0.54 & 0.54 & 1.361 & 1.361 & 0.0 \\
\cline { 2 - 9 } & $1 p_{3 / 2}$ & 50.468 & 15.0 & 0.54 & 0.54 & 1.361 & 1.361 & 0.0 \\
\cline { 2 - 9 } & $1 p_{1 / 2}$ & 51.248 & 15.0 & 0.54 & 0.54 & 1.361 & 1.361 & 0.0 \\
\cline { 2 - 9 } & $1 d_{5 / 2}$ & 47.620 & 15.0 & 0.54 & 0.54 & 1.361 & 1.361 & 0.0 \\
\cline { 2 - 9 } & $2 s_{1 / 2}$ & 50.722 & 15.0 & 0.54 & 0.54 & 1.361 & 1.361 & 0.0 \\
\cline { 2 - 9 } & $1 d_{3 / 2}$ & 49.603 & 15.0 & 0.54 & 0.54 & 1.361 & 1.361 & 0.0 \\
\hline \hline \multirow{3}{*}{$p$} & $1 s_{1 / 2}$ & 55.301 & 15.0 & 0.53 & 0.53 & 1.313 & 1.313 & 1.313 \\
\cline { 2 - 9 } & $1 p_{3 / 2}$ & 53.740 & 15.0 & 0.53 & 0.53 & 1.313 & 1.313 & 1.313 \\
\cline { 2 - 9 } & $1 p_{1 / 2}$ & 54.060 & 15.0 & 0.53 & 0.53 & 1.313 & 1.313 & 1.313 \\
\cline { 2 - 9 } & $1 d_{5 / 2}$ & 49.615 & 15.0 & 0.53 & 0.53 & 1.313 & 1.313 & 1.313 \\
\cline { 2 - 8 } & $2 s_{1 / 2}$ & 52.198 & 15.0 & 0.53 & 0.53 & 1.313 & 1.313 & 1.313 \\
\cline { 2 - 8 } & $1 d_{3 / 2}$ & 52.222 & 15.0 & 0.53 & 0.53 & 1.313 & 1.313 & 1.313 \\
\hline \hline
\end{tabular}

Table (2)

The WS parameters, $U_{0}, U_{s . o}, a_{0}, a_{s . o}, r_{0}, r_{S . o}$, and $R_{C}$ for ${ }^{48}$ Ca nucleus.

\begin{tabular}{|c|c|c|c|c|c|c|c|c|}
\hline${ }^{48} \mathrm{Ca}$ & $n l_{j}$ & $U_{0}$ & $U_{\text {s.o. }}$ & $a_{0}$ & $a_{\text {s.o. }}$ & $r_{0}$ & $r_{\text {S.o. }}$ & $\boldsymbol{R}_{C}$ \\
\hline \multirow{7}{*}{$n$} & $1 s_{1 / 2}$ & 49.352 & 15.0 & 0.5 & 0.5 & 1.242 & 1.242 & 0.0 \\
\hline & $1 p_{3 / 2}$ & 48.286 & 15.0 & 0.5 & 0.5 & 1.242 & 1.242 & 0.0 \\
\hline & $1 p_{1 / 2}$ & 49.996 & 15.0 & 0.5 & 0.5 & 1.242 & 1.242 & 0.0 \\
\hline & $1 d_{5 / 2}$ & 46.463 & 15.0 & 0.5 & 0.5 & 1.242 & 1.242 & 0.0 \\
\hline & $2 s_{1 / 2}$ & 44.561 & 15.0 & 0.5 & 0.5 & 1.242 & 1.242 & 0.0 \\
\hline & $1 d_{3 / 2}$ & 47.291 & 15.0 & 0.5 & 0.5 & 1.242 & 1.242 & 0.0 \\
\hline & $1 f_{7 / 2}$ & 44.422 & 15.0 & 0.5 & 0.5 & 1.242 & 1.242 & 0.0 \\
\hline \multirow{7}{*}{$p$} & $1 s_{1 / 2}$ & 57.953 & 15.0 & 0.54 & 0.54 & 1.284 & 1.284 & 1.284 \\
\hline & $1 p_{3 / 2}$ & 56.616 & 15.0 & 0.54 & 0.54 & 1.284 & 1.284 & 1.284 \\
\hline & $1 p_{1 / 2}$ & 57.789 & 15.0 & 0.54 & 0.54 & 1.284 & 1.284 & 1.284 \\
\hline & $1 d_{5 / 2}$ & 54.579 & 15.0 & 0.54 & 0.54 & 1.284 & 1.284 & 1.284 \\
\hline & $2 s_{1 / 2}$ & 56.580 & 15.0 & 0.54 & 0.54 & 1.284 & 1.284 & 1.284 \\
\hline & $1 d_{3 / 2}$ & 58.442 & 15.0 & 0.54 & 0.54 & 1.284 & 1.284 & 1.284 \\
\hline & $1 f_{7 / 2}$ & 52.052 & 15.0 & 0.54 & 0.54 & 1.284 & 1.284 & 1.284 \\
\hline
\end{tabular}


Table (3)

The calculated $\left(E_{\text {cal }}\right)$ and experimental $\left(E_{\text {exp. }}\right)$ single nucleon binding energies for different subshells for ${ }^{40}$ Ca nucleus.

\begin{tabular}{|c||c||c|}
\hline${ }^{40} \mathbf{C a}$ & $\mathbf{n l}_{\mathbf{j}}$ & $\mathbf{E}_{\mathbf{W S}}=\mathbf{E}_{\text {EXP. [19] }}$ \\
\hline \hline \multirow{4}{*}{$\mathrm{n}$} & $1 \mathrm{~s}_{1 / 2}$ & 42.57 \\
\cline { 2 - 3 } & $1 \mathrm{p}_{3 / 2}$ & 32.42 \\
\cline { 2 - 3 } & $1 \mathrm{p}_{1 / 2}$ & 29.8 \\
\cline { 2 - 3 } & $1 \mathrm{~d}_{5 / 2}$ & 21.6 \\
\cline { 2 - 3 } & $2 \mathrm{~s}_{1 / 2}$ & 18.2 \\
\cline { 2 - 3 } & $1 \mathrm{~d}_{3 / 2}$ & 15.6 \\
\hline \multirow{4}{*}{$\mathrm{p}$} & $1 \mathrm{~s}_{1 / 2}$ & 36.18 \\
\cline { 2 - 3 } & $1 \mathrm{p}_{3 / 2}$ & 26.62 \\
\cline { 2 - 3 } & $1 \mathrm{p}_{1 / 2}$ & 23.14 \\
\cline { 2 - 3 } & $1 \mathrm{~d}_{5 / 2}$ & 14.73 \\
\cline { 2 - 3 } & $2 \mathrm{~s}_{1 / 2}$ & 10.33 \\
\cline { 2 - 3 } & $1 \mathrm{~d}_{3 / 2}$ & 8.3 \\
\hline
\end{tabular}

Table (4)

The calculated $\left(E_{\text {cal }}\right)$ and experimental $\left(E_{\text {exp. }}\right)$ single nucleon binding energies for different subshells for ${ }^{48}$ Ca nucleus.

\begin{tabular}{||c||c||c||}
\hline${ }^{48} \mathbf{C a}$ & $\boldsymbol{n l}_{\boldsymbol{j}}$ & $\boldsymbol{E}_{\boldsymbol{W S}}=\boldsymbol{E}_{\boldsymbol{E X P} \boldsymbol{P} .}[\mathbf{1 9 ]}$ \\
\hline \hline \multirow{4}{*}{$n$} & $1 s_{1 / 2}$ & 39.19 \\
\cline { 2 - 3 } & $1 p_{3 / 2}$ & 30.11 \\
\cline { 2 - 3 } & $1 p_{1 / 2}$ & 28.06 \\
\cline { 2 - 3 } & $1 d_{5 / 2}$ & 20.11 \\
\cline { 2 - 3 } & $2 s_{1 / 2}$ & 12.55 \\
\cline { 2 - 3 } & $1 d_{3 / 2}$ & 12.53 \\
\cline { 2 - 3 } & $1 f_{7 / 2}$ & 9.95 \\
\hline \hline \multirow{4}{*}{$p$} & $1 s_{1 / 2}$ & 39.70 \\
\cline { 2 - 3 } & $1 p_{3 / 2}$ & 30.56 \\
\cline { 2 - 3 } & $1 p_{1 / 2}$ & 28.36 \\
\cline { 2 - 3 } & $1 d_{5 / 2}$ & 20.34 \\
\cline { 2 - 3 } & $2 s_{1 / 2}$ & 15.81 \\
\cline { 2 - 3 } & $1 d_{3 / 2}$ & 16.17 \\
\cline { 2 - 3 } & $1 f_{7 / 2}$ & 9.63 \\
\hline \hline
\end{tabular}

In Table (5), the results of the calculated rms charge, matter, proton and neutron radii are presented for ${ }^{40} \mathrm{Ca}$ and ${ }^{48} \mathrm{Ca}$. The calculated results of the rms charge radii for both ${ }^{40} \mathrm{Ca}$ and ${ }^{48} \mathrm{Ca}$ are in excellent agreement with experimental data using both WS and $\mathrm{HO}$ potentials. The calculated results of the $\mathrm{rms}$ proton radii for ${ }^{40} \mathrm{Ca}$ showed good agreement with experimental data using both WS and $\mathrm{HO}$ potentials while the results for ${ }^{48} \mathrm{Ca}$ is overestimated in WS and slightly overestimated in $\mathrm{HO}$ potential. For the calculated $\mathrm{rms}$ neutron radii, the results for both ${ }^{40} \mathrm{Ca}$ and ${ }^{48} \mathrm{Ca}$ are in excellent agreement with experimental data using $\mathrm{HO}$ while there is an overestimation in WS results for both ${ }^{40} \mathrm{Ca}$ and ${ }^{48} \mathrm{Ca}$. Finally, the results of the calculated rms matter radii showed good agreement with experimental data for ${ }^{40} \mathrm{Ca}$ using WS potential while the result is underestimated in $\mathrm{HO}$ potential. For ${ }^{48} \mathrm{Ca}$, the result obtained with the $\mathrm{HO}$ potential is underestimated and slightly underestimated with that of WS potential.

The calculated CDDs for ${ }^{40} \mathrm{Ca}$ and ${ }^{48} \mathrm{Ca}$ are shown in Figs.(1a) and (2b), respectively. The solid and dashed curves represent the calculated CDDs using WS and $\mathrm{HO}$ radial wave functions, respectively. The dotted curves represent the experimental data [20] taken from the model-independent FourierBessel analysis for both ${ }^{40} \mathrm{Ca}$ and ${ }^{48} \mathrm{Ca}$. It is clear from both figures that the result of WS is better representing the data than those of $\mathrm{HO}$. 
Table (5)

The calculated charge $\left\langle r^{2}\right\rangle_{c h}^{1 / 2}$, matter $\left\langle r^{2}\right\rangle_{m}^{1 / 2}$, proton $\left\langle r^{2}\right\rangle_{p}^{1 / 2}$, and neutron $\left\langle r^{2}\right\rangle_{n}^{1 / 2}$ rms radii in Fermi's ( $\mathrm{fm}$ ) with corresponding available experimental data.

\begin{tabular}{|c|c|c|c|c|c|c|c|c|}
\hline nucleus & $\begin{array}{c}\text { Calculated } \\
\qquad\left\langle r^{2}\right\rangle_{c h}^{1 / 2}\end{array}$ & $\underset{\left\langle r^{2}\right\rangle_{c h}^{1 / 2}}{\operatorname{Exp} .}$ & $\begin{array}{c}\text { Calculated } \\
\left\langle r^{2}\right\rangle_{p}^{1 / 2}\end{array}$ & $\begin{array}{c}\text { Exp. } \\
\left\langle r^{2}\right\rangle_{p}^{1 / 2} \\
{[21]}\end{array}$ & $\begin{array}{c}\text { Calculated } \\
\left\langle r^{2}\right\rangle_{n}^{1 / 2}\end{array}$ & $\begin{array}{c}\text { Exp. } \\
\left\langle r^{2}\right\rangle_{n}^{1 / 2}[21]\end{array}$ & $\begin{array}{c}\text { Calculated } \\
\left\langle r^{2}\right\rangle_{m}^{1 / 2}\end{array}$ & $\underset{\left\langle\boldsymbol{r}^{2}\right\rangle_{m}^{\mathbf{1 / 2}}}{\text { Exp. }}\left[\left[^{2} 2\right]\right.$ \\
\hline \multirow{2}{*}{${ }^{40} \mathrm{Ca}$} & WS: 3.451 & \multirow{2}{*}{$3.450(10)$} & WS: 3.361 & \multirow{2}{*}{$3.36(3)$} & WS: 3.391 & \multirow{2}{*}{$3.31(3)$} & WS: 3.376 & \multirow{2}{*}{3.37} \\
\hline & HO: 3.450 & & HO: 3.358 & & HO: 3.310 & & HO: 3.334 & \\
\hline \multirow{2}{*}{${ }^{48} \mathrm{Ca}$} & WS: 3.451 & \multirow{2}{*}{$3.451(9)$} & WS: 3.361 & \multirow{2}{*}{$3.32(3)$} & WS: 3.600 & \multirow{2}{*}{$3.43(2)$} & WS: 3.503 & \multirow{2}{*}{$3.58(4)$} \\
\hline & HO: 3.451 & & HO: 3.359 & & HO: 3.430 & & HO: 3.400 & \\
\hline
\end{tabular}

The results of the calculated charge form factors are illustrated in Figs. (2a and b) for ${ }^{40} \mathrm{Ca}$ and ${ }^{48} \mathrm{Ca}$, respectively. The calculated results using the radial wave functions of WS and $\mathrm{HO}$ potentials are indicated by solid and dashed curves, respectively. The dotted curves in Fig. (2) are the experimental data and taken from [23]. Again, it is obvious from Figs. (2a and $b)$ that the calculated results using WS radial wave functions depicted by solid curves are better representing the data than that of $\mathrm{HO}$ potential depicted by dashed curves, where the radial wave functions of $\mathrm{HO}$ potential failed to predict the existence of the third diffraction minima for both ${ }^{40} \mathrm{Ca}$ and ${ }^{48} \mathrm{Ca}$.
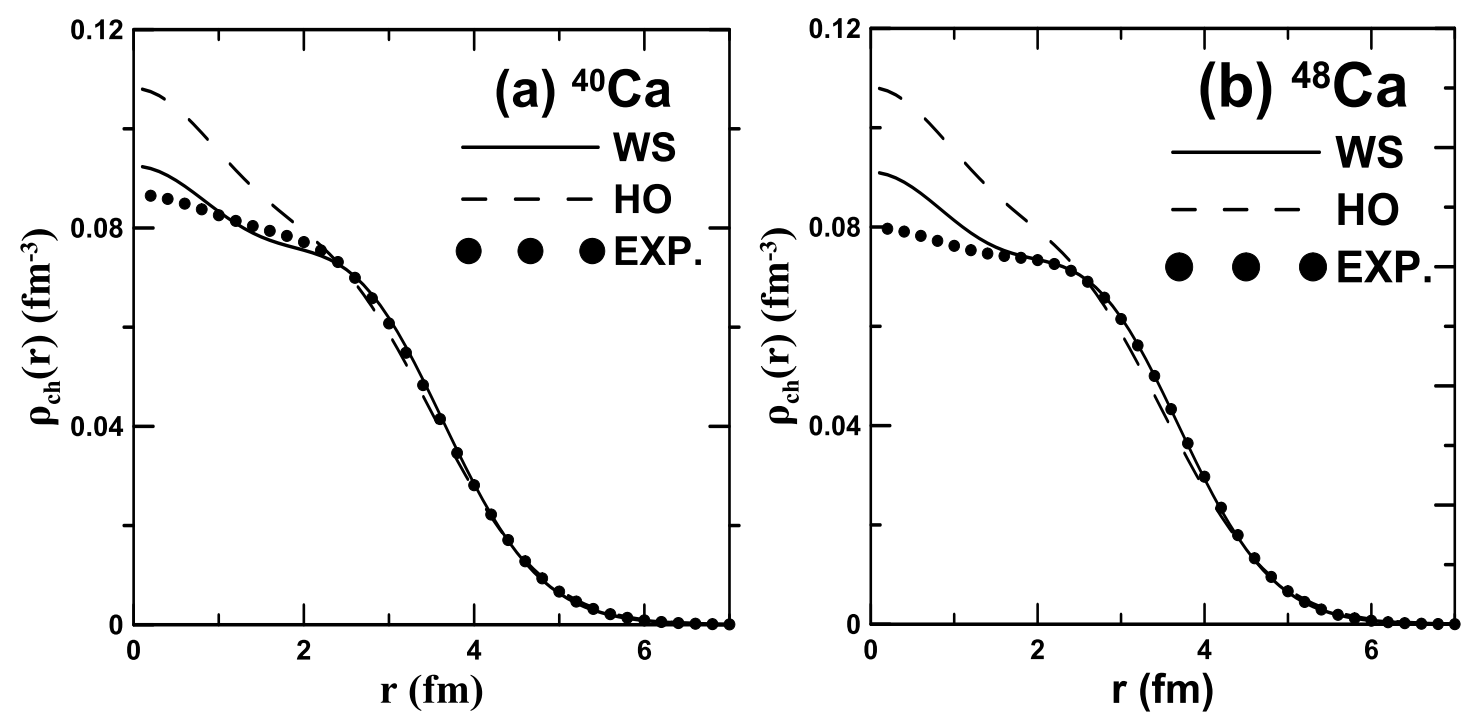

Fig.(1): CDDs for ${ }^{40} \mathrm{Ca}$ (a) and ${ }^{48} \mathrm{Ca}(\mathrm{b})$ obtained by WS (solid curve) and HO (dashed curve) potentials. The experimental data for both are denoted by filled dotted circles and taken from [20]. 

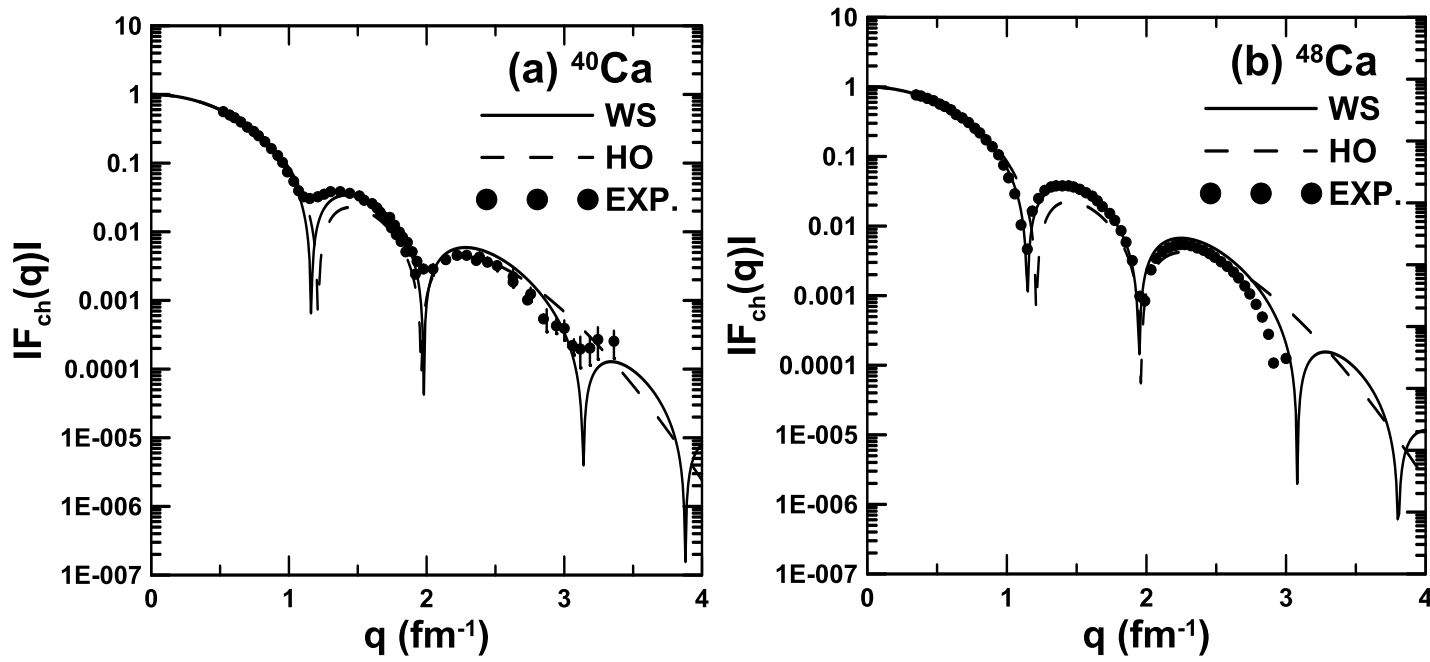

Fig.(2): Charge form factors for ${ }^{40} \mathrm{Ca}\left(\right.$ a) and ${ }^{48} \mathrm{Ca}(\mathrm{b})$ calculated by WS (solid curve) and $\mathrm{HO}$ (dashed curve) potentials. The experimental data are denoted by filled dotted circles and taken for ${ }^{40} \mathrm{Ca}$ and ${ }^{48} \mathrm{Ca}$ from [23].

\section{Conclusions}

The nuclear CDD, elastic charge form factors, and rms charge, proton, neutron and matter radii besides single nucleon binding energies are investigated for both ${ }^{40} \mathrm{Ca}$ and ${ }^{48} \mathrm{Ca}$ using the radial wave functions of WS and $\mathrm{HO}$ potentials. It is found an excellent agreement between the calculated $r m s$ charge radii and experimental data for both isotopes in both WS and $\mathrm{HO}$ potentials. The results of the calculated proton $r m s$ radii for ${ }^{40} \mathrm{Ca}$ are found to be in good agreement with experiment data using both WS and $\mathrm{HO}$ potentials while the results for ${ }^{48} \mathrm{Ca}$ showed an overestimation in WS potential and slight overestimation in $\mathrm{HO}$ potential in comparison with experimental data. The results for the calculated neutron $\mathrm{rms}$ radii are found to be well predicted in $\mathrm{HO}$ potential for both ${ }^{40} \mathrm{Ca}$ and ${ }^{48} \mathrm{Ca}$, while there is overestimation in WS results for both isotopes. The calculated $\mathrm{rms}$ matter radii showed good agreement with experimental data for ${ }^{40} \mathrm{Ca}$ using WS potential while the result is underestimated in $\mathrm{HO}$ potential. For ${ }^{48} \mathrm{Ca}$, the results using $\mathrm{HO}$ potential is underestimated and slightly underestimated using WS potential. The calculated CDDs using WS radial wave functions are in good agreement with experimental data for both ${ }^{40} \mathrm{Ca}$ and ${ }^{48} \mathrm{Ca}$. Regarding the calculated charge form factors, the results in WS potential is in much better agreement with experimental data using WS where the results using $\mathrm{HO}$ potential are completely failed to predict the existence the third diffraction minimum for both isotopes under study.

\section{References}

[1] Batty C. J., Friedman E., Gils H. J., and Rebel H., "Experimental methods for studying nuclear density distributions", Advances in Nuclear Physics, 19, 1-188, 1989.

[2] G. R.Satchler and Love W. G., "Folding model potentials from realistic interactions for heavy-ion scattering”, Physics Reports, 55, 183-254, 1979.

[3] Elton L. R. B. and Swift A., "Singleparticle potentials and wave functions in the 1p and 2s-1d shells", Nuclear Physics A. 94, 52-72, 1967.

[4] Gibson B. F. Goldberg A., and Weiss M. S., "Single-particle ground-state model of ${ }^{4} \mathrm{He}$ ", Nuclear Physics A. 111, 321-330, 1968.

[5] Gamba S., Ricco G., And Rottigni G., “A phenomenological Woods-Saxon potential for p-shell nuclei", Nuclear Physics A. 213, 383-396, 1973.

[6] B. A. Brown, Massen S. E., and Hodgson P. E., "Proton and neutron density distributions for $A=16-58$ nuclei", Journal of Physics G. 5, 1655-1698, 1979.

[7] Owen A. S., Brown B. A., and Hodgson P. E., "Nucleon orbit radii in tin isotopes", Journal of Physics G: Nuclear Physics, 7, 1057-1061, 1981. 
[8] Street J., Brown B. A., and Hodgson P. E., "Nuclear matter distributions". Journal of Physics G: Nuclear Physics, 8, 839-850, 1982.

[9] Brown B. A., Massen S. E., Escudero J. I., Hodgson P. E., Madurga G., and Vinas J., "The charge and matter distributions of ${ }^{208} \mathrm{~Pb}$ ", Journal of Physics G: Nuclear Physics, 9, 423-441, 1983.

[10] Lojewski Z., Nerlo-Pomorska B., and Pomorski K., "Mean square radii of nuclei calculated with the Woods-Saxon potential", Physical Review C. 51(2), 601605, 1995.

[11] Lojewski Z. and Dudek J., "Various parametrizations of the Woods-Saxon potential", Acta Physica Polonica B. 29 (12), 407-417, 1998.

[12] Schwierz N., Wiedenhover I., and Volya A., "Parametrization of the WoodsSaxon potential for shell-model calculations", arxiv: nucl-th/0709.3525v1, 1-19, 21 Sep 2007.

[13] Fatah A. H., "Calculation of the eigenvalues for Wood-Saxon's potential by using Numerov method", Advances in theoretical and applied mechanics, 5, 2331, 2012.

[14] Ridha A. R., "Study of charge density distributions, elastic charge form factors and root-mean square radii for ${ }^{4} \mathrm{He},{ }^{12} \mathrm{C}$ and ${ }^{16} \mathrm{O}$ nuclei using Woods-Saxon and harmonic-oscillator potentials", Iraqi Journal of physics, 14, 42-50, 2016.

[15] Peter Ring and Peter Schuck, "The Nuclear Many-Body Problem", SpringerVerlag, 1981.

[16] Antonov A. N., Hodgson P. E., and Petkov I. Zh., "Nucleon Momentum and Density Distributions in Nuclei", Clarendon Press, Oxford, 1988.

[17] Elton L. R. B., "Nuclear Sizes", Oxford University Press, 1961.

[18] Brussard P. J. and Glaudemans P. W. M., "Shell-Model Application in Nuclear Spectroscopy", North-Holland, Amesterdam, 1977.

[19] Lederer C. M. and Shirley V. S., "Table of Isotopes, $7^{\text {th }}$ ed.", John Wiley and Sons, New York, 1978.

[20] De Vries H., De Jager C. W., and De Vries C., "Nuclear charge-density- distribution parameters from elastic electron scattering", Atomic Data and Nuclear Data Tables 36, 495-536, 1987.

[21] Gibbs W. R. and Dedonder J.-P., "Neutron radii of the calcium isotopes", Physical Review C. 46, 1825-1833, 1992.

[22] Batty C. J., Friedman E., Gils H. J., and Rebel H., "Experimental methods for studying nuclear density distributions", Advances in Nuclear Physics, 19, 1-188, 1989.

[23] Emrich H. J., Ph.D. thesis, University of Mainz, 1983. 